\title{
EXTENSIONS IN CERTAIN TOPOLOGICAL ALGEBRAIC CATEGORIES
}

\author{
BY \\ ROBERT C. BUSBY
}

\begin{abstract}
Categories which we call "sufficiently algebraic" are defined, and for certain objects $A$ (called faithful) in such categories, and arbitrary objects $C$, we partially order the sets Ext $(C, A)$ of extensions of $A$ by $C$. We prove that the maximal elements in Ext $(C, A)$ (with respect to this ordering) are in bijective correspondence with the morphisms from $C$ to a canonical object $O(A)$. If the short five lemma holds in the category, all extensions are maximal and therefore obtained in this way.

As an application we compute extensions in certain categories of topological rings. In particular we investigate the possible extensions of one group algebra (of a locally compact group) by another in the category of Banach algebras with norm decreasing homomorphisms, and using some analytic tools we give conditions for the splitting of such extensions. Previous results of the author on extensions of $C^{*}$-algebras are also included in this theory as a special case.
\end{abstract}

Introduction. In a previous paper [1], the author considered extensions in the category of $C^{*}$-algebras. In the present paper we give a general categorical setting for results from [1] and we apply the results to some categories which are useful in analysis and substantially less well behaved than the category of $C^{*}$-algebras.

Specifically in $\S 1$ we define, for certain categories, objects which we call faithful. We show that if $A$ is a faithful object and $C$ is any object in the category, there is a bijective correspondence between $\operatorname{Hom}(C, O(A))$ and all "greatest" elements in certain subsets of Ext $(C, A) . O(A)$ is an object whose nature is explicitly exhibited. If the short five lemma from category theory holds (see [8, Proposition 21.1]), which it does not in some categories useful for analysis, then we get all of Ext $(C, A)$ in the correspondence.

In $\$ 2$ we give some examples to illustrate the main result. The theory requires little of a category and in fact seems useful only in categories which are not too well behaved. For example, abelian categories trivially have the properties which we will require, but they would seem to contain no faithful objects. The best examples are various categories of topological rings.

In $\S 3$, we apply the theory to the problem of extending one group algebra (of a locally compact group) by another or by a measure algebra. Our very complete

Received by the editors December 12, 1969.

AMS 1969 subject classifications. Primary 4650; Secondary 4680, 1810.

Key words and phrases. Extensions, faithful rings, double centralizers, group algebras, splitting extensions. 
knowledge of norm decreasing homomorphisms of group algebras due to Greenleaf [3] enables us to get results in these cases.

1. Extensions in certain categories. We will need some definitions and results from category theory and for all these we will refer to Mitchell [8]. In particular we assume familiarity with the definitions of kernel, cokernel, pullback, zero object, and intersections in categories.

Definition 1.1 . We will say that a category $\mathscr{A}$ is sufficiently algebraic (SA) if the following three properties hold:

(i) $\mathscr{A}$ has a zero object and pullbacks (thus also kernels and finite intersections, see [8, Chapter 1]).

(ii) If

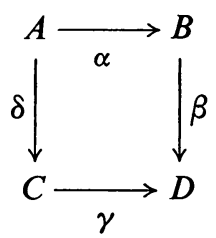

is a pullback diagram and $\gamma$ is the cokernel of its kernel then so is $\alpha$.

(iii) If $\alpha$ is a map such that $\operatorname{ker}(\alpha)=0$ then $\alpha$ is a monomorphism.

Definition 1.2. Let $A$ be an SA category. A short exact sequence is a diagram $E: A \stackrel{\alpha}{\rightarrow} B \stackrel{\beta}{\rightarrow} C$ in $\mathscr{A}$ such that $\alpha$ is the kernel of $\beta$ and $\beta$ is the cokernel of $\alpha$. If we wish to leave $\alpha$ and $\beta$ unspecified, we will denote the sequence by $E: A \succ B \rightarrow C$ as in Mac Lane [7]. Throughout the rest of this section we will be dealing with a fixed SA category $\mathscr{A}$.

Definition 1.3. Let $E_{i}: A_{i} \gg B_{i} \rightarrow C_{i}(i=1,2)$ be short exact sequences. By a morphism between $E_{1}$ and $E_{2}$ we shall mean a triple $(\alpha, \beta, \gamma)$ of maps $\alpha: A_{1} \rightarrow A_{2}, \beta: B_{1} \rightarrow B_{2}, \gamma: C_{1} \rightarrow C_{2}$ making the following diagram commutative:

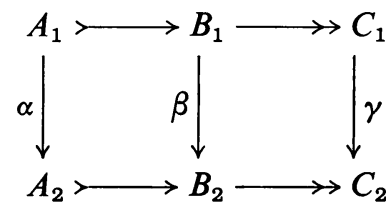

Definition 1.4. Let $E_{i}: A \succ B_{i} \rightarrow C(i=1,2)$ be short exact sequences. We will say that

(a) $E_{1} \leqq E_{2}$ if there is a morphism $(1, \beta, 1)$ from $E_{1}$ to $E_{2}$ (1 represents the appropriate identity map).

(b) $E_{1} \cong E_{2}$ (equivalence) if there is a morphism $(1, \beta, 1)$ from $E_{1}$ to $E_{2}$ with $\beta$ an isomorphism.

It is trivial to show that $\leqq$ is reflexive and transitive, $\cong$ is an equivalence relation, and the relation $\leqq$ depends only on the equivalence classes of the elements. We will denote the equivalence class of an exact sequence $E$ by $[E]$. 
Let Ext $(C, A)$ denote the set of all equivalence classes of exact sequences $A \longmapsto B \rightarrow C$ for fixed $A$ and $C$. The above discussion shows that Ext $(C, A)$ has a natural preorder relation which we will also denote by $\leqq$. The short five lemma holds if and only if this preorder relation is trivial. ([E $E_{1} \leqq\left[E_{2}\right] \Leftrightarrow\left[E_{1}\right]=\left[E_{2}\right]$.) We call Ext $(C, A)$ the set of extensions of $C$ by $A$.

Lemma 1.1. Let $E: A \stackrel{\alpha}{\rightarrow} B \stackrel{\beta}{\rightarrow} C$ be exact and let $\gamma \in \operatorname{Hom}\left(C^{\prime}, C\right)$. Then

(i) There is an object $B^{\prime} \in \mathscr{A}$, an exact sequence $E^{\prime}: A \stackrel{\alpha^{\prime}}{\longrightarrow} B^{\prime} \stackrel{\beta^{\prime}}{\longrightarrow} C^{\prime}$, and a morphism $(1, \theta, \gamma)$ from $E^{\prime}$ to $E$.

(ii) If $P(E, \gamma)$ represents the set of all classes $\left[E^{\prime}\right]$ in $\operatorname{Ext}\left(C^{\prime}, A\right)$ for which there is a morphism $(1, \theta, \gamma)$ from $E^{\prime}$ to $E$, then $P(E, \gamma)$ depends only on $[E]$ and $\gamma$.

(iii) There is a canonical greatest element (under $\leqq)$ in $P(E, \gamma)$ which we will denote by $[E]_{y}$.

Proof. (i) Let

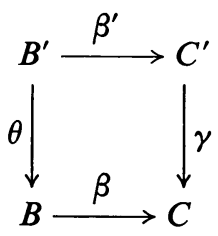

be the pullback diagram associated with the pair $(\beta, \gamma)$. (This exists by property (i) of an SA category.)

By hypothesis on $E, \beta$ is the cokernel of its kernel and so by property (ii) of $\mathscr{A}$, $\beta^{\prime}$ must be the cokernel of its kernel. Let $\eta: A \rightarrow C^{\prime}$ be the zero morphism and let $\alpha: A \rightarrow B$ be as given in the hypothesis. Then $\beta \alpha=0=\gamma \eta$ and, by the defining property of pullbacks, there is a unique map $\alpha^{\prime}: A \rightarrow B^{\prime}$ such that $\beta^{\prime} \alpha^{\prime}=\eta$ and $\theta \alpha^{\prime}=\alpha$. We therefore have a sequence $A \stackrel{\alpha^{\prime}}{\longrightarrow} B^{\prime} \stackrel{\beta^{\prime}}{\longrightarrow} C^{\prime}$ with $\beta^{\prime} \alpha^{\prime}$ the zero map. We will show that $A \stackrel{\alpha^{\prime}}{\longrightarrow} B^{\prime}$ is the kernel of $B^{\prime} \stackrel{\beta^{\prime}}{\longrightarrow} C^{\prime}$.

Let $K \stackrel{\mu}{\rightarrow} B^{\prime}$ be a monomorphism with $\beta^{\prime} \mu=0$. Then $0=\beta^{\prime} \mu=\gamma \beta^{\prime} \mu=\beta \theta \mu$. Since $A \stackrel{\alpha}{\rightarrow} B$ is the kernel of $\beta$, there must be a map $\rho: K \rightarrow A$ with $\theta \mu=\alpha \rho=\theta \alpha^{\prime} \rho$. Also $\beta^{\prime} \alpha^{\prime} \rho=0=\beta^{\prime} \mu$. Since $\beta^{\prime} \mu=\beta^{\prime} \alpha^{\prime} \rho$ and $\theta \mu=\theta \alpha^{\prime} \rho$, the uniqueness assertion in the definition of pullback guarantees that $\mu=\alpha^{\prime} \rho$ and this in turn proves that $A \stackrel{\alpha^{\prime}}{\longrightarrow} B^{\prime}$ is the kernel of $\beta^{\prime}$. Since $\beta^{\prime}$ is then the cokernel of $\alpha^{\prime}$, we see that $E^{\prime}: A \stackrel{\alpha^{\prime}}{\longrightarrow} B^{\prime} \stackrel{\beta^{\prime}}{\longrightarrow} C^{\prime}$ is exact and we have also shown that $(1, \theta, \gamma)$ is a morphism from $E^{\prime}$ to $E$.

(ii) This assertion is straightforward.

(iii) We will show that for the sequence $E^{\prime}$ constructed above, $\left[E^{\prime}\right]$ is a greatest element in $P(E, \gamma)$. [ $\left.E^{\prime}\right]$ will be $[E]_{\gamma}$.

Let $E^{\prime \prime}: A \stackrel{\alpha^{\prime \prime}}{\longrightarrow} B^{\prime \prime} \stackrel{\beta^{\prime \prime}}{\longrightarrow} C^{\prime}$ be in $P(E, \gamma)$ and let $\left(1, \theta^{\prime \prime}, \gamma\right)$ be a morphism from $E^{\prime \prime}$ to $E$. Since $\beta \theta^{\prime \prime}=\gamma \beta^{\prime \prime}$ and $E^{\prime}$ comes from a pullback diagram, there must be a unique map $\delta: B^{\prime \prime} \rightarrow B^{\prime}$ such that $\beta^{\prime \prime}=\beta^{\prime} \delta$ and $\theta^{\prime \prime}=\theta^{\prime} \delta$. 
Consider the diagram

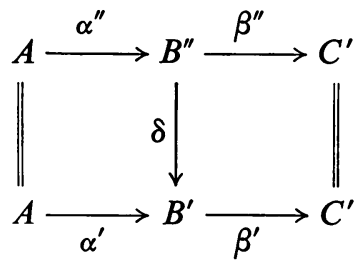

(where the vertical maps are identities).

We claim that this diagram is commutative. We already know that $\beta^{\prime \prime}=\beta^{\prime} \delta$. Now $\delta \alpha^{\prime \prime}$ is such that $\beta^{\prime}\left(\delta \alpha^{\prime \prime}\right)=\beta^{\prime \prime} \alpha^{\prime \prime}=0$ and $\theta^{\prime}\left(\delta \alpha^{\prime \prime}\right)=\theta^{\prime \prime} \alpha^{\prime \prime}=\alpha$. The uniqueness assertion concerning pullbacks then requires that $\delta \alpha^{\prime \prime}=\alpha^{\prime}$ and we are done.

We now define faithful objects in an SA category. The following definition is due essentially to Dauns [2].

Definition 1.5. We say that an object $M$ in an SA category $\mathscr{A}$ is a maximal container for an object $A$ in $\mathscr{A}$ if the following conditions hold:

(a) There is a monomorphism $\alpha_{0}: A \rightarrow M$ defining $A$ as a normal subobject of $M$.

(b) $A$ is essential in $M$, i.e. if $\stackrel{\beta}{\rightarrow} M$ is a normal subobject of $M$ such that $C \cap A=0$ then $C=0$ (the zero object).

(c) If $\beta: A \rightarrow B$ defines $A$ as a normal subobject of $B$, then there is a unique map $\theta_{B}: B \rightarrow M$ such that $\theta_{B} \beta=\alpha_{0}$.

Definition 1.6. We say that an object $A$ is faithful if $A$ has a maximal container.

We remark that a maximal container can be trivially shown to be unique up to isomorphism if it exists and so we will denote the maximal container of a faithful object $A$ by $M(A)$.We will let $O(A)$ represent the quotient object of $A \stackrel{\alpha_{0}}{\longrightarrow} M(A)$, i.e. there is a map $M(A) \stackrel{\pi}{\rightarrow} O(A)$ which is the cokernel of $\alpha_{0}$. We will also denote the corresponding short exact sequence by $E_{0} .\left[E_{0}\right]$ is uniquely determined by $A$.

LEMMA 1.2. Let $\gamma$ be any map in $\operatorname{Hom}(C, O(A))$. Then there is precisely one greatest element in $P\left(E_{0}, \gamma\right)$.

Proof. Let $E: A \stackrel{\alpha}{\rightarrow} B \stackrel{\beta}{\rightarrow} C$ represent $\left[E_{0}\right]_{\gamma}$ and let $(1, \theta, \gamma)$ be a morphism from $E$ to $E_{0}$ such that

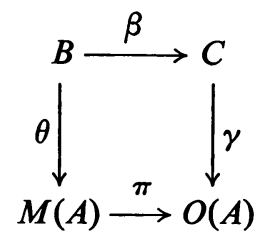

is a pullback diagram (this is possible from Lemma 1.1). Let $E^{\prime}: A \stackrel{\alpha^{\prime}}{\longrightarrow} B^{\prime} \stackrel{\beta^{\prime}}{\longrightarrow} C$ be such that $\left[E^{\prime}\right]$ is another greatest element in $P\left(E_{0}, \gamma\right)$, and let $\left(1, \theta^{\prime}, \gamma\right)$ be the corresponding morphism. Then $\left[E^{\prime}\right] \leqq[E]$ and $[E] \leqq\left[E^{\prime}\right]$. We can therefore find maps $\rho: B \rightarrow B^{\prime}$ and $\rho^{\prime}: B^{\prime} \rightarrow B$ such that $(1, \rho, 1)$ maps $E$ to $E^{\prime}$ and $\left(1, \rho^{\prime}, 1\right)$ maps $E^{\prime}$ to $E$. 
First we claim that $\rho^{\prime}$ is a monomorphism. In fact if $K \stackrel{\delta}{\rightarrow} B^{\prime}$ is the kernel of $\rho^{\prime}$, then $\rho^{\prime} \delta=0=\beta \rho^{\prime} \delta=\beta^{\prime} \delta$ and since $\alpha^{\prime}$ is the kernel of $\beta^{\prime}$, there must be a map $\xi: K \rightarrow A$ with $\delta=\alpha^{\prime} \xi$. Then $\rho^{\prime} \delta=\rho^{\prime} \alpha^{\prime} \xi=\alpha \xi=0$. Since $\alpha$ is a monomorphism $\xi=0$ and so $\delta=0$. Now condition (iii) in the definition of SA guarantees that $\rho^{\prime}$ is a monomorphism.

We further note that $\rho^{\prime} \rho: B \rightarrow B$ is such that $\beta\left(\rho^{\prime} \rho\right)=\beta$. Also since $\theta \rho^{\prime} \rho: B \rightarrow M(A)$ has the property that $\theta \rho^{\prime} \rho \alpha=\theta \alpha=\alpha_{0}$, the definition of $M(A)$ yields $\theta \rho^{\prime} \rho=\theta$. Finally the fact that $B$ is a pullback yields $\rho^{\prime} \rho=1_{B}$ (identity).

Now $\rho^{\prime}\left(\rho \rho^{\prime}\right)=\left(\rho^{\prime} \rho\right) \rho^{\prime}=1_{B} \rho^{\prime}=\rho^{\prime} 1_{B}$.

Since $\rho^{\prime}$ is a monomorphism, $\rho \rho^{\prime}=1_{B}$, and so $\rho$ and $\rho^{\prime}$ are isomorphisms and $[E]=\left[E^{\prime}\right]$.

The canonical sequence has an additional property which manifests itself in the following lemma.

Lemma 1.3. Let $\gamma_{1}, \gamma_{2} \in \operatorname{Hom}(C, O(A)), \gamma_{1} \neq \gamma_{2}$. Then $P\left(E_{0}, \gamma_{1}\right) \cap P\left(E_{0}, \gamma_{2}\right)=\varnothing$.

Proof. Suppose $[E](E: A \stackrel{\alpha}{\rightarrow} B \stackrel{\beta}{\rightarrow} C)$ is in the intersection. Let $\left(1, \theta_{1}, \gamma_{1}\right)$ and $\left(1, \theta_{2}, \gamma_{2}\right)$ be the corresponding morphisms to $E_{0}$. Then $\theta_{i}: B \rightarrow M(A)(i=1,2)$ have the property that $\theta_{i} \alpha=\alpha_{0}$. By definition of $M(A), \theta_{1}=\theta_{2}$. Then $\gamma_{1} \beta=\pi \theta_{1}$ $=\pi \theta_{2}=\gamma_{2} \beta$ and since $\beta$ is an epimorphism, $\gamma_{1}=\gamma_{2}$.

Lemma 1.4. Let $E: A \stackrel{\alpha}{\rightarrow} B \stackrel{\beta}{\rightarrow} C$ be exact. Then for some $\gamma \in \operatorname{Hom}(C, O(A))$, $[E] \in P\left(E_{0}, \gamma\right)$.

Proof. There is a map $\theta: B \rightarrow M(A)$ such that $\theta \alpha=\alpha_{0}$. Consider the following diagram

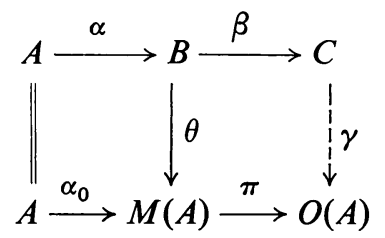

which commutes. Then $\pi \theta \alpha=0$ and since $\beta$ is the cokernel of $\alpha$, the diagram can be uniquely filled in (by the broken arrow) by a map $\gamma$ so that it is commutative.

We now know by Lemmas 1.3 and 1.4 that the sets $\left\{P\left(E_{0}, \gamma\right) \mid \gamma \in \operatorname{Hom}(C, O(A))\right\}$ form a partition of $\operatorname{Ext}(C, A)$. Lemma 1.2 tells us that there is at most one greatest element in each $P\left(E_{0}, \gamma\right)$, while Lemma 1.1 says that there is at least one and it is given explicitly as $\left[E_{0}\right]_{\gamma}$. Denoting the set of all these greatest elements by $G$ Ext $(C, A)$ we have proved the main theorem of this section:

THEOREM 1.1. The map from $\operatorname{Hom}(C, O(A))$ to $G \operatorname{Ext}(C, A)$ given by $\gamma \rightarrow\left[E_{0}\right]_{\gamma}$ is a bijection.

CoRollary 1.1. Suppose $\mathscr{A}$ is an SA category in which the short five lemma holds, $A$ is faithful in $\mathscr{A}, E_{0}: A \stackrel{\alpha_{0}}{\longrightarrow} M(A) \stackrel{\pi}{\longrightarrow} O(A)$ is the canonical sequence, and $C \in \mathscr{A}$, then $\gamma \rightarrow\left[E_{0}\right]_{\gamma}$ is a bijection between $\operatorname{Hom}(C, O(A))$ and $\operatorname{Ext}(C, A)$. 
Definition 1.7. Let $E: A \stackrel{\alpha}{\rightarrow} B \stackrel{\beta}{\rightarrow} C$ be an exact sequence in $\mathscr{A}$. We say that

(a) $E$ is split or semidirect if there is a map $\delta: C \rightarrow B$ such that $\rho \delta=1$ (the identity on $C$ ).

(b) $E$ is essential if $A$ is essential in the sense of Definition 1.5(b).

It is not hard to show that these definitions depend only on the class $[E]$ in Ext $(C, A)$ and we will apply the corresponding adjectives to such classes.

Now suppose that $\gamma \in \operatorname{Hom}(C, O(A))$ for $A, C \in \mathscr{A}$ and $A$ faithful. Let $[E]$ correspond to $\gamma$ as in Theorem 1.1 where $E$ is the sequence $A \stackrel{\alpha}{\rightarrow} B \stackrel{\beta}{\rightarrow} C$.

THEOREM 1.2. (a) $[E]$ is semidirect $\Leftrightarrow \exists \rho: C \rightarrow M(A)$ with $\pi \rho=\gamma$.

(b) Let $\mathscr{A}$ have the following properties:

(i) Every kernel is a normal subobject.

(ii) Suppose $A$ is faithful with maximal container $M(A), A \succ B$ defines $A$ as a normal subobject of $B$, and

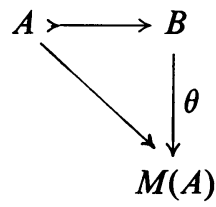

is the diagram which the definition of maximal container guarantees to exist. Let $A^{\prime} \longrightarrow B$ be a normal subobject of $B$ with $A^{\prime} \cap A=0$ then $A^{\prime} \subset \operatorname{ker} \theta$.

Under these conditions $[E]$ is essential if and only if $\gamma$ is injective.

Proof. (a) We may assume that there is a pullback diagram

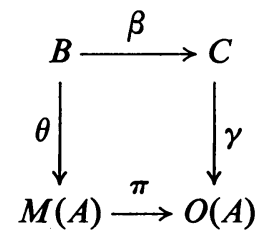

where $B$ occurs in $E$. If $[E]$ is semidirect and $\delta: C \rightarrow B$ such that $\beta \delta=1$, let $\rho=\theta \delta$. Then $\rho: C \rightarrow M(A)$ and $\pi \rho=\pi \theta \delta=\gamma \beta \delta=\gamma$.

Conversely suppose $\exists \rho: C \rightarrow M(A)$ with $\pi \rho=\gamma$. Since $B$ is a pullback there is exactly one map $\delta: C \rightarrow B$ such that $\theta \delta=\rho$ and $\beta \delta=1$. In particular [E] is split.

(b) Consider the entire diagram

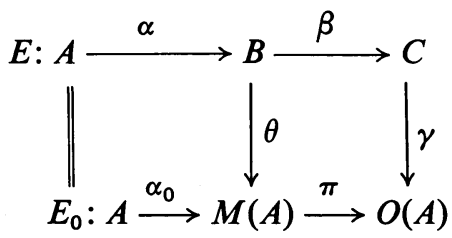

The right-hand square is a pullback, and the left-hand square may be regarded as coming from the definition of $M(A)$ once $E$ is given. 
First look at $D=\operatorname{ker} \theta$ and let $j: D \rightarrow B$ be the canonical injection. If $Z=A \cap D$ (which exists in $\mathscr{A}$ ) there must be maps $p: Z \rightarrow A ; q: Z \rightarrow D ; r: Z \rightarrow B$ such that $\alpha p=r=j q$. Then $\theta r=\theta \alpha p=\alpha_{0} p$ and $\theta r=\theta j q=0=\alpha_{0} 0$. Since $\alpha_{0}$ is a monomorphism, $p=0$. Then $r=\alpha p=0$ so $z$ is 0 and $A \cap \operatorname{ker} \theta=0$. This computation tells us that if $A$ is essential in $B, \operatorname{ker} \theta=0$. Condition (ii) above tells us that if $\operatorname{ker} \theta=0, A$ is essential in $B$. To complete our proof, we show that $\operatorname{ker} \theta=0 \Leftrightarrow \operatorname{ker} \gamma=0$.

In fact first assume that ker $\theta=0$ and let $A^{\prime}=\beta^{-1}(\operatorname{ker} \gamma)$ (and $A^{\prime} \stackrel{t}{\rightarrow} B$ be the canonical injection). Then $\pi \theta t=\gamma \beta t=0$ and $\exists r: A^{\prime} \rightarrow A$ such that $\alpha_{0} r=\theta t=\theta \alpha r$. By condition (iii) for $\mathscr{A}, \theta$ is a monomorphism and $t=\alpha$. Thus $\beta t=0=\operatorname{ker} \gamma$.

Conversely suppose ker $\gamma=0$, and let $A^{\prime} \stackrel{\xi}{\rightarrow} B$ be ker $\theta$. Then $\pi \theta \xi=0=\gamma \beta \xi$ and since ker $\gamma=0, \beta \xi=0$. Then there is a map $s: A^{\prime} \rightarrow A$ such that $\alpha s=\xi$, and thus $A^{\prime} \subset A \cap \operatorname{ker} \theta$ which is zero by a computation performed above. Thus ker $\theta=A^{\prime}=0$. Condition (iii) on $\mathscr{A}$ tells us that $\gamma$ is injective $\Leftrightarrow \operatorname{ker} \gamma=0$ and so we have shown that $E$ is essential $\Leftrightarrow \gamma$ is injective.

We remark finally that $[E]$ is trivial $(B$ is the direct sum of $A$ and $B) \Leftrightarrow \gamma=0$. This is proved in a straightforward manner and we omit the proof.

2. Examples. (a) Let $\mathscr{A}$ be the category of abstract groups and homomorphisms. It is easy to show that $G$ is faithful if and only if $G$ has trivial center, in which case $M(G)=$ Aut $(G)$, the group of all automorphisms of $G . O(G)$ is then the group of "outer automorphisms" of $G$. Since the short five lemma holds in $\mathscr{A}$ and $\mathscr{A}$ is easily shown to be an SA category, our main result shows that there is a bijective correspondence between $\operatorname{Hom}\left(G^{\prime}, O(G)\right)$ and $\operatorname{Ext}\left(G^{\prime}, G\right)$ for any group $G^{\prime}$. This result is essentially well known, see [7, Chapter iv, $\S 9$, Theorem 9.1].

(b) Let $\mathscr{A}$ be the category of associative rings. $A \in \mathscr{A}$ is customarily called faithful if for $x \in A,(x a=0 \forall a \in A) \Rightarrow x=0$ and $(a x=0 \forall a \in A) \Rightarrow x=0$. If $A$ is faithful in this sense then $A$ is faithful in our sense. In this case $M(A)$ is the ring of double centralizers of B. E. Johnson (see [5]). Again $\mathscr{A}$ is SA and the short five lemma is true, so that if $A$ is faithful and $C$ is any ring, Ext $(C, A)$ is in bijective correspondence with all ring homomorphisms $\operatorname{Hom}(C, O(A))$.

This example may be modified by requiring $\mathscr{A}$ to be the category of real or complex algebras, or commutative rings, or commutative algebras or *-algebras, etc. and one will get all algebra extensions or commutative extensions, etc., for the faithful objects. A trivial and amusing consequence of this is the (well-known) result that all extensions of one commutative faithful ring by another commutative ring are commutative and similarly for algebras.

We remark now that it is possible to consider many different categories of topological rings. To have faithful objects in our sense, it will be necessary to have the double centralizer algebra with some suitable topology belong to the category. We will consider only a few possibilities. The interested reader can consult [5] and [6] for information about double centralizers with various topologies and then formulate categories to which our results apply. 
(c) Let $\mathscr{A}$ be the category of $C^{*}$-algebras. This category is SA, every object is faithful (here the double centralizer algebra of $A$ is also a $C^{*}$-algebra) and the main theorem applies. We recover the results of [1] which were proved by more explicit constructions there. The main theorem is put to use in $[1, \S 7]$ to show how in some cases we may explicitly write down all extensions.

(d) Let $\mathscr{A}$ be the category of "Banachable algebras" and continuous linear maps, where $A \in \mathscr{A}$ is a topological algebra which has a compatible norm in which it is complete. We are in effect looking at Banach algebras and concerning ourselves only with the topology and continuous homomorphisms, not with the norm. The short five lemma holds in this category due to the interior mapping principle and we can therefore construct all extensions of such faithful algebras $\mathrm{A}$, both algebraically and topologically from elements in $\operatorname{Hom}(C, O(A))$.

If we are interested in the norm, not just the topology, we must consider a different category. We do this next and discover our first interesting category in which the short five lemma fails to hold.

(e) Let $\mathscr{A}$ be the category of Banach algebras and norm decreasing homomorphisms. The unusual categorical nature of $\mathscr{A}$ justifies our examining $\mathscr{A}$ much more closely than we did previous categories.

First we remark that for $A \stackrel{\alpha}{\rightarrow} B \stackrel{\beta}{\rightarrow} C$ to be a short exact sequence in our sense, $\alpha$ must be an isometry and $C$ must have the quotient norm under $\beta$. The former follows from the fact that $\alpha$ is the kernel of $\beta$ categorically, and so in particular $A$ must have the minimal norm for which $\alpha$ is norm decreasing. Similar remarks using the definition of cokernel show that $C$ must have the maximal norm for which $\beta$ is norm decreasing, and this is the quotient norm.

$\mathscr{A}$ is an SA category and this also needs some proof.

Evidently condition (iii) holds. In connection with (i) note that if

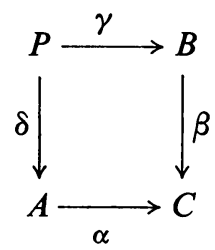

is a diagram then we define the pullback algebraically to be

$$
P=\{(a, b) \in A \times B \mid \alpha(a)=\beta(b)\}
$$

We must then provide a norm for this algebra $P$, and if the pullback conditions are to hold it must be a minimal norm compatible with having $\gamma$ and $\delta$ be norm decreasing ( $\gamma$ and $\delta$ are the usual projection maps restricted to $P$ ). This norm is the $\max$ norm, $\|(a, b)\|=\max (\|a\|,\|b\|)$, and so pullbacks exist. 
Now if $\alpha$ is the cokernel of its kernel then $\alpha$ is onto and $C$ has the quotient norm. $\gamma$ is then onto and $B$ is algebraically the cokernel of $\operatorname{ker}(\gamma)$. We show that $B$ has the quotient norm. Let $y_{0} \in B$.

$$
\begin{aligned}
\gamma^{-1}\left(y_{0}\right) & =\left\{\left(x, y_{0}\right) \mid \alpha(x)=\beta\left(y_{0}\right)\right\} \\
& =\left\{\left(x, y_{0}\right) \mid \beta\left(y_{0}\right)=z=\alpha\left(x_{0}\right), x=x_{0}+s, s \in \text { ker } \alpha\right\}
\end{aligned}
$$

now if $\left(x, y_{0}\right) \in \gamma^{-1}\left(y_{0}\right),\left\|\left(x, y_{0}\right)\right\|=\max \left(\|x\|,\left\|y_{0}\right\|\right) \geqq\left\|y_{0}\right\|$. On the other hand

$$
\begin{aligned}
\left\|y_{0}\right\| & \geqq\left\|\beta\left(y_{0}\right)\right\|=\left\|\alpha\left(x_{0}\right)\right\| \\
& =\inf _{s \in \operatorname{ker} \alpha}\left\|x_{0}+s\right\|=\inf _{\left(x, y_{0}\right) \in \gamma^{-1}\left(y_{0}\right)}\|x\| .
\end{aligned}
$$

So $\left\|y_{0}\right\|=\inf _{\left(x, y_{0}\right) \in \gamma^{-1}\left(y_{0}\right)}\left\|\left(x, y_{0}\right)\right\|$ and $B$ has the quotient norm. Hence $\mathscr{A}$ is an SA category. If $A$ is faithful in the algebraic sense, and $m \in M(A)$, the algebraic double centralizer algebra, we define $\|m\|_{1}=\sup _{\|a\| \leqq 1}\|m a\|,\|m\|_{2}=\sup _{\| a ! \mid \leqq 1}\|a m\|$, and $\|m\|=\max \left(\|m\|_{1},\|m\|_{2}\right)$; then it is not hard to verify that $M(A)$ with this norm satisfies the definition of maximal container and so $A$ is categorically faithful. Now the short five lemma does not hold in this category. A trivial example is provided by letting $A$ and $C$ be $R^{1}, B_{1}$ be $R^{2}$ with the norm $\|(x, y)\|_{1}=\|x\|+\|y\|$, and $B_{2}$ be $R^{2}$ with the norm $\|(x, y)\|_{2}=\max (\|x\|,\|y\|)$. Then the following diagram is commutative:

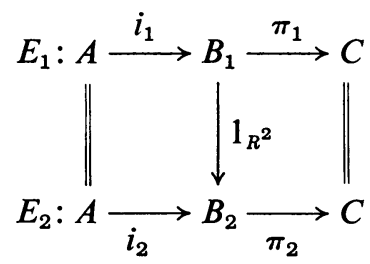

Here $i_{j}$ and $\pi_{j}(j=1,2)$ are the usual injection and projection maps and $1_{R^{2}}$ is the identity map which is an algebraic and topological isomorphism. Clearly $E_{1}$ and $E_{2}$ are short exact sequences in $\mathscr{A}$ and $\left(1,1_{R^{2}}, 1\right)$ is a morphism between them, and since $1_{R^{2}}$ is in this case not an isometry, the short five lemma fails. In spite of this our main theorem now applies and gives us a bijective correspondence between all norm decreasing homomorphisms from any $C \in \mathscr{A}$ to $O(A)$, and those extensions $A \stackrel{\alpha}{\rightarrow} B \stackrel{\beta}{\rightarrow} C$ such that

(1) $\alpha$ is an isometry.

(2) The norm of $C$ is the quotient norm through $\beta$ of the norm in $B$.

(3) The norm of $B$ is the minimal norm compatible with the above-mentioned properties and the fact that $B$ is a Banach algebra.

Note that $B$ is algebraically and topologically uniquely determined by the corresponding element of Hom $(C, O(A))$. The norm, however, is not unique, but a minimal such exists and we must choose it. Then $B$ can be canonically constructed (norm included) from the corresponding element in $\operatorname{Hom}(C, O(A))$. If we compare this example with the previous one, we see that if $\gamma: C \rightarrow O(A)$ is continuous but 
not norm decreasing then the corresponding extension is an exact sequence in the previous category but not the present one. In particular if $A \rightarrow B \rightarrow C$ is the sequence, $C$ will have the quotient topology but not the quotient norm, and no renorming of $B$ compatible with all other properties of the extension will result in $C$ having the quotient norm and $A$ having the subspace norm.

This category and that of the previous example can each be modified by requiring the algebras to be *-algebras. The results then obtained are similar to those described above.

We remark finally that each of the examples given here satisfies the conditions of Theorem 1.2.6 and so if $[E] \in \operatorname{Ext}(C, A)$ corresponds to $\gamma \in \operatorname{Hom}(C, A)$, then $[E]$ is essential if and only if $\gamma$ is injective.

We will give only a brief sketch of how one proves this fact.

It is clear that every kernel is normal in all these examples (even though in the last category epimorphisms are not necessarily cokernels of their kernels).

If we are in a group based category, $A$ is normal in $B, A^{\prime}$ is normal in $B, A \cap A^{\prime}$ $=0$, and $\theta: B \rightarrow M(A)$, then if $g \in A^{\prime}, x \in A$, we would have $\theta(g) x=g^{-1} x g$. The group element $x^{-1} g^{-1} x g \in A \cap A^{1}=0$ so $x g=g x$ and $\theta(g)$ is the identity. Thus $A^{\prime} \subset \operatorname{ker} \theta$. Similar considerations hold in the algebra based categories.

3. Extensions of group algebras. Among the most widely studied Banach algebras are the group algebras of locally compact groups. Thanks to Greenleaf and Cohen before him, we have a very precise description of the norm decreasing homomorphisms from one group algebra to another. It is interesting to see what Banach algebras are extensions of one group algebra by another, and in the present section we apply the results of $\S 1$ to this problem. In the rest of this section an extension of $C$ by $A$ will mean an element of $G$ Ext $(C, A)$, that is we will specify the norm. To get the other elements of $\operatorname{Ext}(C, A)$ one simply changes to equivalent norms.

Again we will work in the category $\mathscr{A}$ of Banach algebras and norm decreasing homomorphisms. If $A$ is the group algebra of a locally compact group $G$, then $A$ is a faithful object in $\mathscr{A}$ and the double centralizer algebra $M(A)$ is nothing else but the algebra $M(G)$ of all bounded regular Borel measures on $G$ with convolution as product and the total variation as norm (see [6]). $L^{1}(G)$ can be considered to be a norm-closed two-sided ideal in $M(G)$ by identifying it with those measures absolutely continuous with respect to Haar measure.

Now suppose $L^{1}(G)$ and $L^{1}(F)$ are objects in $\mathscr{A}$. Then our main results tell us that we can get all extensions of $L^{1}(F)$ by $L^{1}(G)$ by considering norm decreasing homomorphisms of $L^{1}(F)$ into $O(G)$. We can, by Theorem 1.2(a), get all split or semidirect extensions by considering all norm decreasing homomorphisms of $L^{1}(F)$ into $M(G)$. The latter have been studied in detail by Greenleaf in [3] and we will use his results to find all split extensions. We will then show that in many cases all extensions are in fact split. 
Let us summarize Greenleaf's results. He has shown first of all that an arbitrary subgroup of the unit ball in $M(G)$ is constructed as follows: Let $H_{0}$ be a subgroup in $G$. Let $K$ be a compact subgroup of $G$ with $K \subset H_{0}$, and let $\rho$ be a character on $K$ such that

(1) $K$ and $K_{0}=\operatorname{ker} \rho$ are both normal in $H_{0}$.

(a) $K / K_{0}$ is central in $H_{0} / K_{0}$.

Let $\Omega$ be any subgroup of $T^{1} \times G\left(T^{1}\right.$ is the unit circle) with

$$
H_{0}=\left\{g \in G:(\alpha, g) \in \Omega \text { for some } \alpha \in T^{1}\right\} .
$$

Let $m_{K}$ be normalized Haar measure in $K$.

Then $\Gamma=\left\{\alpha \delta_{g} * \rho m:(\alpha, g) \in \Omega\right\}$ is a subgroup of the unit ball in $M(G)$ and $H_{0}=\bigcup\{s(\mu): \mu \in \Gamma\}(s(\mu)$ being the support of $\mu)$. The unit of $\Gamma$ is $\rho m_{K}$. Furthermore all subgroups of the unit sphere are constructed in this manner.

In particular $\left\{\delta_{g} * m_{K} \mid K\right.$ compact in $G, g \in H_{0}, K$ normal in $\left.H_{0}\right\}$ is a group in the unit sphere for any subgroup $H_{0}$ (not necessarily closed) of $G$. This is a group of translates of normalized Haar measure of a compact subgroup. Now suppose $\Gamma$ is the subgroup described above. Let $\Omega_{0}=\left\{(\alpha, g) \in \Omega \mid \alpha \delta_{g} * \rho m_{K}=\rho m_{K}\right\}$. Give the quotient group $\Omega / \Omega_{0}$ the subspace topology considering it as a subspace of the right coset space $\left(T^{1} \times G\right) / \Omega_{0}$, where the latter has the quotient topology. Let $\theta: F \rightarrow \Omega / \Omega_{0}$ be a continuous onto homomorphism. Then $\theta$ generates a norm decreasing homomorphism which we now describe. If $K(G)$ denotes the continuous functions with compact support on $G, f \in K(G)$, and $(\alpha, g) \in T^{1} \times G$, we define $\hat{f}(\alpha, g)$ to be $\left(\alpha \delta_{g} * \rho m_{K}\right)(f)$. Then $\hat{f}$ is continuous on $T^{1} \times G$ and constant on right cosets of $\Omega_{0}$, and so corresponds to a function $\psi(f)$ in $K\left(T^{1} \times G / \Omega_{0}\right)$. We then let $\hat{\theta}(f) \in K(F)$ be defined by $\hat{\theta}(f)(x)=\psi(f)(\theta(x))$ for all $x \in F$. Finally if $\mu \in M(F)$, define $\check{\theta}(\mu) \in M(G)$ by $\check{\theta}(\mu)(f)=\mu(\hat{\theta}(f))$, for all $f \in K(G)$. Greenleaf has shown that $\check{\theta}$ is a norm decreasing homomorphism from $M(F)$ to $M(G)$ and every norm decreasing homomorphism from $L^{1}(F)$ to $M(G)$ arises by restricting such a $\check{\theta}$ to $L^{1}(F)$. In particular, norm decreasing homomorphisms from $L^{1}(F)$ to $M(G)$ always extends uniquely to $M(F)$ and such extensions are norm decreasing.

THEOREM 3.1. Let $F$ and $G$ be locally compact groups. Then

(i) There is a one-to-one correspondence between split extensions of $L^{1}(F)$ by $L^{1}(G)$ and split extensions of $M(F)$ by $L^{1}(G)$.

(ii) Let $\left\{H_{0}, K, \rho, \Omega, \Omega_{0}\right\}=\mathscr{S}$ be a system as described above. Let $\theta: F \rightarrow \Omega / \Omega_{0}$ be a continuous epimorphism and let $\check{\theta}: M(F) \rightarrow M(G)$ be as previously constructed. Then $B=\left\{(a+\check{\theta}(\mu), \mu) \mid a \in L^{1}(G), \mu \in M(F)\right\}$ is a Banach algebra with pointwise operations and the sup norm. If $a \in L^{1}(G)$, then $\alpha(a)=(a, 0) \in B$, and if $(a+\check{\theta}(\mu), \mu)=$ $z \in B$, then $\beta(z)=\mu \in M(F)$. Furthermore, $L^{1}(G) \stackrel{\alpha}{\rightarrow} B \stackrel{\beta}{\rightarrow} M(F)$ is a split extension and all such extensions arise in this way depending only on $\mathscr{S}$ and $\theta$.

(iii) Using the notation of (ii), we let $B_{0} \subset B$ be defined by

$$
B_{0}=\left\{(a+\check{\theta}(f), f) \mid a \in L^{1}(G), f \in L^{1}(F)\right\}
$$


then we get in an obvious way a sequence $L^{1}(G) \stackrel{\alpha_{0}}{\longrightarrow} B_{0} \stackrel{\beta_{0}}{\longrightarrow} L^{1}(F)$ which is the most general split extension of $L^{1}(F)$ by $L^{1}(G)$.

Proof. (i) follows from the one-to-one correspondence between maps from $L^{1}(F)$ to $M(G)$ and from $M(F)$ to $M(G)$ in $\mathscr{A}$. (ii) and (iii) follow immediately from our main theorem (Theorem 1.1) and Corollary 1.1, Greenleaf's characterization of norm decreasing homomorphisms, and the particular form of the pullback in the category $\mathscr{A}$.

We can now explicitly construct all split extensions if we know the group structure of $G$ and $F$ and can compute certain group homomorphisms. We would like to have more information about when an extension is split. We begin by investigating $M(G)$ more closely.

Let $S(G)$ be the set of measures singular with respect to Haar measure (this definition does not depend on which Haar measure is used). Then $S(G)$ is a normclosed subspace of $M(G)$, complementary to $L^{1}(G) . S(G)$ is not a subalgebra of $M(G)$, but it has certain multiplicative properties and is complementary to $L^{1}(G)$ in a very special way. From now on the convolution of a measure $\mu$ and a measure $\nu$ on any group will be written simply $\mu \nu$.

Lemma 3.1. Let $s \in S(G)$ and $a \in L^{1}(G)$. Then $\|s+a\|=\|s\|+\|a\|$.

Proof. See [4, Theorem 19.20(iii)].

LEMMA 3.2. If $\mu, \nu \in M(G),\|\mu\| \leqq 1,\|\nu\| \leqq 1, \mu \nu=a+s$ with $a \in L^{1}(G)$ and $s \in S(G)$ and if $\|s\|=1$, then $\mu \in S(G), \nu \in S(G)$, and $\mu \nu=s$.

Proof. Let $\mu=b+t, \nu=c+r ; b, c \in L^{1}(G)$ and $t, r \in S(G)$. Then $a+s=\mu \nu=$ $b c+b r+t c+t r=a^{\prime}+\operatorname{tr}$ with $a^{\prime} \in L^{1}(G)$. Then $1 \geqq\|\mu\|=\|b\|+\|t\| \geqq\|t\|$ and similarly $1 \geqq\|c\|+\|r\| \geqq\|r\|$ and thus $1 \geqq\|t\|\|r\| \geqq\|t r\|=\left\|s+\left(a-a^{\prime}\right)\right\|=\|s\|+\left\|a-a^{\prime}\right\|=$ $1+\left\|a-a^{\prime}\right\| \geqq 1$. We see from this that $a-a^{\prime}=0$ and so $\operatorname{tr}=s$. Then $1=\|s\| \leqq$ $\|t\|\|r\| \leqq 1$ and so $\|t\|=1$ and $\|r\|=1$. Since $1 \geqq\|\mu\|=\|b\|+\|t\|=\|b\|+1$ we have $b=0$ and similarly $c=0$.

The lemma now follows.

CoRollaRy 3.1. If $\mu \in M(G)$ is invertible and $\|\mu\| \leqq 1,\left\|\mu^{-1}\right\| \leqq 1$, then $\mu$ and $\mu^{-1}$ are singular.

CoRollary 3.2. If $\|\mu\| \leqq 1,\|\nu\| \leqq 1, \mu, \nu \in S(G)$ and $\mu \nu-1 \in L^{1}(G)$ (1 is the identity in $M(G)$ ), then $\mu=\nu^{-1}$.

CoROllaRy 3.3. If $\mu \in S(G),\|\mu\| \leqq 1$ and $\mu^{2}-\mu \in L^{1}(G)$, then $\mu^{2}=\mu$ and $\mu$ is an idempotent in $S(G)$.

Proof. Since $\|\mu\| \leqq 1,\left\|\mu^{2}\right\| \leqq\|\mu\|^{2} \leqq\|\mu\| \leqq\|\mu\|+\|a\|=\|\mu+a\|=\left\|\mu^{2}\right\|\left(a=\mu^{2}-\mu\right)$. Thus $\|\mu\|=\|\mu\|^{2}$ and so $\|\mu\|=1$. The corollary then follows.

Now let $S^{\prime}(G)$ be the group of invertible elements $\mu$ in $S(G)$ such that $\|\mu\| \leqq 1$ and $\left\|\mu^{-1}\right\| \leqq 1$. Let $O^{\prime}(G)$ be the corresponding group in $O(G)=M(G) / L^{1}(G)$ and let $\pi: M(G) \rightarrow O(G)$ be the natural projection. Finally let $\rho=\pi \mid S(G)$. Then 
Proposition 3.1.

(a) $\rho$ is an isometry,

(b) $\rho\left(S^{\prime}(G)\right)=O^{\prime}(G)$,

(c) $\rho(\mu)$ is idempotent of norm $1, \Leftrightarrow \mu$ is idempotent of norm 1 .

Proof. (a) $\|\rho(\mu)\|=\inf _{a \in L^{1}(G)}\|\mu+a\|=\inf _{a \in L^{1}(G)}\|\mu\|+\|a\|=\|\mu\|$.

(b) Clearly $\rho\left(S^{\prime}(G)\right) \subset O^{\prime}(G)$.

If $\rho(\mu) \in O^{\prime}(G)$ then $\|\mu\| \leqq 1$ and $\exists \nu$ such that $\|\nu\| \leqq 1$ and $\rho(\mu) \rho(\nu)=1$. Thus $\mu \nu-1 \in L^{1}(G)$ and Corollary 3.2 yields the result.

(c) $\Leftarrow$ is clear. If $\|\rho(\mu)\| \leqq 1$ and $\rho(\mu)$ is idempotent then $\mu^{2}-\mu \in L^{1}(G)$ and $\|\mu\| \leqq 1$. The result then follows from Corollary 3.3.

We will now prove two propositions which will give us information about when extensions are split. The first proposition deals with the problem of extending a norm decreasing homomorphism mapping $L^{1}(F)$ to $O(G)$ to a certain subset of $M(F)$, and the second deals with norm decreasing monomorphisms.

Let $\phi: L^{1}(F) \rightarrow O(G)$ be a norm decreasing homomorphism, where $F$ and $G$ are any locally compact groups. Let $\Sigma_{1}=\left\{\delta_{x} \mid x \in F\right\}$. It is known that $\Sigma_{1}$ is contained in the set of invertible elements $\mu$ in $M(F)$ such that $\|\mu\| \leqq 1$ and $\left\|\mu^{-1}\right\| \leqq 1$, and in fact the latter set consists entirely of elements of $\Sigma_{1}$ multiplied by complex numbers of absolute value 1 [9, Theorem 3]. Let $\xi: O(G) \rightarrow S(G)$ be the inverse of $\rho$ (notation as above), and let $\Phi=\xi \phi$. Then

Proposition 3.2. $\Phi$ extends to a map (also written $\Phi)$ from the set $L^{1}(F) \cup \Sigma_{1}$ to $M(G)$ such that

(i) If $\mu, \nu \in \Sigma_{1}, \Phi(\mu) \Phi(\nu)=\Phi(\mu \nu)$.

(ii) If $\mu \in \Sigma_{1}, x \in L^{1}(F), \Phi(\mu) \Phi(x)=\Phi(\mu x)$.

(iii) If $\left(e_{i}\right)_{i \in I}$ is a norm-one two-sided approximate identity in $L^{1}(F)$ and $\mu \in \Sigma_{1}$, $\Phi(\mu)=\lim _{i \rightarrow \infty} \Phi\left(\mu e_{i}\right)$ in the weak-* topology of $M(G)$.

Proof. The proof of this proposition is a modification of the proof of [3, Theorem 4.1.1]. In order to facilitate matters, we will refer to the above proof and simply indicate the necessary alterations.

Let $\left(e_{i}\right)$ be a norm-one two-sided approximate identity in $L^{1}(F)$. The set $\left\{\Phi\left(e_{i}\right)\right\}$ is bounded and therefore has a weak-* limit point $\lambda$. Greenleaf has shown in [3, Lemma 4.1.2] that if $\Phi$ is a norm decreasing homomorphism then $\lambda$ is the unique limit point of the set $\left\{\Phi\left(e_{i}\right)\right\}$. The only property of use in his proof which our map $\Phi$ lacks is the property that $\Phi\left(e_{i} f\right)=\Phi\left(e_{i}\right) \Phi(f)$ for $f \in L^{1}(F)$. It is easy to see that this may be replaced in the proof by $\lim _{i \rightarrow \infty}\left\|\Phi\left(e_{i}\right) \Phi(f)-\Phi\left(e_{i} f\right)\right\|=0$. We assert that this holds for our $\Phi$. In fact let $\Phi\left(e_{i}\right) \Phi(f)=\Phi\left(e_{i} f\right)+a(i, f)$ where $a(i, f) \in L^{1}(G)$. Let $b(i, f)=\Phi\left(e_{i} f\right)-\Phi(f) \in S(G)$. Then $\Phi\left(e_{i}\right) \Phi(f)=\Phi(f)+b(i, f)+a(i, f)$, and so using Lemma 3.1, we have

$$
\begin{aligned}
\|\Phi(f)\|+\|a(i, f)\| & =\|\Phi(f)+a(i, f)\| \\
& \leqq\left\|\Phi\left(e_{i}\right)\right\|\|\Phi(f)\|+\|b(i, f)\| \leqq\|\Phi(f)\|+\|b(i, f)\| .
\end{aligned}
$$


Then $\|a(i, f)\| \leqq\|b(i, f)\|$ and the latter tends to zero as $i \rightarrow \infty$. This proves our assertion.

We then use this fact in the same way that Greenleaf used Lemma 4.1.2 in the proof of Theorem 4.1.1. Again the property we lack is that if $\mu \in \Sigma_{1}$ and $\mu_{i}$ is a net in $L^{1}(F)$ such that $\mu_{i} f \rightarrow \mu f$ and $f \mu_{i} \rightarrow f \mu$ for all $f \in L^{1}(F)$, then $\Phi\left(\mu_{i} f\right)=\Phi\left(\mu_{i}\right) \Phi(f)$ for all $f \in L^{1}(F)$, and again it will be sufficient to have $\lim _{i \rightarrow \infty}\left\|\Phi\left(\mu_{i}\right) \Phi(f)-\Phi\left(\mu_{i} f\right)\right\| \rightarrow 0$.

If we mimic the similar computation given above, we will see that it goes through provided that $\|\Phi(\mu f)\|=\|\Phi(f)\|, f \in L^{1}(F)$. This is proved below in Lemma 3.3 and so we have proved (iii). (ii) follows from (iii) and the calculation given two lines above. To show (i) let $\mu, \nu \in \Sigma_{1}$ and let $\mu_{i} \in L^{1}(F)$ such that $\mu_{i} x \rightarrow \mu x$ and $x \mu_{i} \rightarrow x \mu \forall x \in L^{1}(F)$. Then $\nu \mu_{i} x \rightarrow \nu \mu x$ etc. and so $\Phi\left(\nu \mu_{i}\right) \rightarrow \Phi(\nu \mu)$. Also $\Phi\left(\mu_{i}\right) \rightarrow \Phi(\mu)$ so $\Phi(v) \Phi\left(\mu_{i}\right) \rightarrow \Phi(\nu) \Phi(\mu)$. Finally (ii) tells us that $\Phi\left(\nu \mu_{i}\right)=\Phi(v) \Phi\left(\mu_{i}\right)$ and so $\Phi(\nu) \Phi(\mu)=\Phi(\nu \mu)$ and (i) is proved.

LemMa 3.3. Let $\mu \in \Sigma_{1}, f \in L^{1}(F)$. Then $\|\Phi(\mu f)\|=\|\Phi(f)\|$.

Proof. It is enough to show that $\|\phi(\mu f)\|=\|\phi(f)\|$ by Proposition 3.1(a). Now let $\left(e_{i}\right)_{i \in I}$ be an approximate identity in $L^{1}(F)$ as before. Then

$$
\|\phi(\mu f)\|=\lim _{i \rightarrow \infty}\left\|\phi\left(e_{i} \mu f\right)\right\| \leqq \limsup _{i \rightarrow \infty}\left\|\phi\left(e_{i} \mu\right)\right\|\|\phi(f)\| \leqq\|\phi(f)\|
$$

since $\phi$ is norm decreasing and $\|\mu\| \leqq 1$.

Since $f=\mu^{-1}(\mu f)$ and $\mu^{-1} \in \Sigma_{1}$, we see that $\|\phi(\mu f)\|=\|\phi(f)\|$.

This completes the lemma and so the proof of Proposition 3.2.

Let $G$ and $F$ be locally compact groups such that $F$ has a compact open normal subgroup $K$. Let $\phi: L^{1}(F) \rightarrow O(G)$ be a norm decreasing monomorphism and let $\xi$ be as above. Then

Proposition 3.3. $\Phi=\xi \phi$ is a norm decreasing monomorphism from $L^{1}(F)$ to $M(G)$ such that $\pi \Phi=\phi$ ( $\pi$ is the projection of $M(G)$ onto $O(G)$ ).

Proof. Let $m$ be the normalized Haar measure of $K$. Since $K$ is open, $m \in L^{1}(F)$, and we know that $m$ is an idempotent of norm one. Consider $L^{1}(K) \subset L^{1}(F)$, and let $x \in L^{1}(K)$. Then $m x=c m$ for some constant which depends on $x$.

If $x \geqq 0$ then $m x=\|x\| m$. If $y=x /\|x\|$, where $x$ is a fixed positive element of $L^{1}(K)$, then $m y=m$ so $\phi(m) \phi(y)=\phi(m)$. Since $\Phi(m) \phi(y)-\Phi(m) \in L^{1}(G)$, Lemma 3.2 tells us that $\Phi(m) \Phi(y)=\Phi(m)$. Notice that since $\phi$ has trivial kernel, $\phi(m)$ is a nonzero idempotent and so $\Phi(m)$ is also a nonzero idempotent and both have norm 1. Let $\sigma$ be supp $(\Phi(m))$. Then $\sigma$ is a compact group. Notice that since $\|\Phi(m)\|=\|\Phi(m y)\| \leqq\|\Phi(m)\|\|\Phi(y)\| \leqq\|\Phi(m)\|$, we have $\|\Phi(y)\|=1$. Putting these facts together we see that $\|\Phi(m)\|\|\Phi(y)\|=1=\|\Phi(m)\|=\|\Phi(m) \Phi(y)\|$ and so [3, Theorem 2.1.1] tells us that if $\delta$ is the support of $\Phi(y)$, then $\sigma \delta \subset \sigma$. Since $\sigma$ is a group, $\delta \subset \sigma$. This must also hold for $x$. If $z \in L^{1}(K)$ then $z=x_{1}-x_{2}$ with $x_{i} \geqq 0$ $(i=1,2)$, so $\Phi(z)=\Phi\left(x_{1}\right)-\Phi\left(x_{2}\right)$. The preceding analysis shows that $\Phi\left(x_{1}\right)$ and $\Phi\left(x_{2}\right)$ both have support within $\sigma$, and so for all $z \in L^{1}(K), \Phi(z)$ has support 
contained in a fixed compact group $\sigma$. Since for any two measures, the support of the convolution is contained in the product of the supports, $\Phi(x) \Phi(y) \in S(G)$ for all $x, y \in L^{1}(K)$. Since we know that $\Phi(x y)$ is the singular part of $\Phi(x) \Phi(y)$, it follows that $\Phi: L^{1}(K) \rightarrow M(G)$ is a homomorphism which by Proposition 3.1 and assumptions on $\phi$ is norm decreasing.

Now suppose that $x \in L^{1}(K), f \in F$, and $y=\delta_{f} * x$. By Proposition 3.2 and results of Greenleaf previously described, we have $\Phi(y)=\Phi\left(\delta_{f}\right) * \Phi(x)=\alpha \delta_{g} * \rho s * \Phi(x)$, where $g \in G$, and $s$ is the Haar measure of the compact subgroup $n=\operatorname{supp}\left(\Phi\left(\delta_{e}\right)\right)$ of $G$. Since $\delta_{e} * m=m$, it follows easily that $n \sigma \subset \sigma$ and so $n \subset \sigma$. Thus the support of $\Phi(y)$ is contained in a coset of $\sigma$.

Let $x \in L^{1}(F)$. Then for each coset $\xi K$ of $K$ in $L^{1}(F), x \mid \xi K$ is in $L^{1}(F)$ and for different cosets $\xi_{1} K$ and $\xi_{2} K, x \mid \xi_{1} K$ and $x \mid \xi_{2} K$ are mutually singular. Thus if $R$ is a complete set of coset representatives in $F$ for cosets of $K, x=\sum_{\xi \in R} x \mid \xi K$, where this series is norm convergent and so all but a countable number of terms are zero. Hence $x=\sum_{n=1}^{\infty} x_{n}$ where each $x_{n}$ has support in a coset of $K$ and the series converges in norm. It follows that $\Phi(x)=\sum_{n=1}^{\infty} \Phi\left(x_{n}\right)$. Similarly if $y \in L^{1}(F)$, $\Phi(y)=\sum_{n=1}^{\infty} \Phi\left(y_{n}\right)$ (with perhaps different cosets of $K$ involved). Then $\Phi(x) \Phi(y)$ is the norm limit of measures

$$
\mu_{N}=\left(\sum_{n=1}^{N} \Phi\left(x_{n}\right)\right)\left(\sum_{n=1}^{N} \Phi\left(y_{n}\right)\right)=\sum_{i, j=1}^{N} \Phi\left(x_{i}\right) \Phi\left(y_{j}\right) .
$$

Since each term $\Phi\left(x_{i}\right) \Phi\left(y_{j}\right)$ has support in some coset of $\sigma$, we see that $\mu_{N}$ is singular $\forall N$, and so $\Phi(x) \Phi(y)$ is singular and as before $\Phi(x) \Phi(y)=\Phi(x y)$. Thus $\Phi$ is a norm decreasing monomorphism and clearly $\pi \Phi=\Phi$. This completes the proof.

Using Propositions 3.2 and 3.3, we can now describe the splitting of certain extensions.

THEOREM 3.2. Let $G$ and $F$ be locally compact groups and let us call any extension in $G \operatorname{Ext}\left(L^{1}(F), L^{1}(G)\right)$ a proper extension of $L^{1}(F)$ by $L^{1}(G)$. Then

(a) If $F$ has a compact open normal subgroup, then all essential proper extensions of $L^{1}(F)$ by $L^{1}(G)$ are split.

(b) If $F$ is discrete, all proper extensions of $L^{1}(F)$ by $L^{1}(G)$ are split.

(c) If $G$ is discrete, all proper extensions of $L^{1}(F)$ by $L^{1}(G)$ are trivial (isomorphic with the direct product).

(d) If $F$ is compact and $G$ has no nontrivial compact subgroups, then again all proper extensions of $L^{1}(F)$ by $L^{1}(G)$ are trivial.

Proof. (a) We remarked earlier that a proper extension in $\mathscr{A}$ is essential if and only if it corresponded to a norm decreasing monomorphism. (a) follows from this, Proposition 3.3, and Theorem 1.2(a).

(b) In the proof of Proposition 3.3, the injectivity of the norm decreasing monomorphism was used only to insure that the Haar measure $m_{K}$ went onto a 
nonzero idempotent. If $F$ is discrete we may take $K$ to be the identity and then $m_{K}$ either goes to a nonzero element or else the entire homomorphism is zero and we get a direct product. Thus (b) follows.

(c) is trivial since in this case $M(G)=L^{1}(G)$ so $O(G)=0$.

(d) If $F$ is compact, and $\phi: L^{1}(F) \rightarrow O(G)$ corresponds to our proper extension, then Proposition 3.2 shows that there is a natural homomorphism $\Phi$ of $F$ onto a subgroup $\Gamma$ of the unit ball in $M(G) . \Gamma=\left\{\alpha \delta_{g} * \rho m_{K} \mid g \in H_{0}, m_{K}\right.$ Haar measure of $K, K$ compact in $G$. Our condition on $G$ implies that $K$ and $\rho$ are trivial. Then $\Gamma=\left\{\alpha \delta_{g}\left|g \in H_{0},\right| \alpha \mid=1,(\alpha, g) \in \Omega\right\}$ where $\Omega \subset T^{1} \times G$ is a subgroup. In this case $\Omega_{0}$ (notation as before) is trivial and so there is a continuous homomorphism of $F$ onto $\Omega$. This implies that $\Omega$ is compact and so $H_{0}$ is compact. Thus $H_{0}=e$ and $\Phi=0$. In this case $\Phi=0$ and the extension is trivial.

\section{REFERENCES}

1. R. C. Busby, Double centralizers and extensions of $C^{*}$-algebras, Trans. Amer. Math. Soc. 132 (1968), 79-99. MR 37 \#770.

2. J. Dauns, Multiplier rings and primitive ideals, Trans. Amer. Math. Soc. 145 (1969), 125-158.

3. F. P. Greenleaf, Characterization of group algebras in terms of their translation operators, Pacific J. Math. 18 (1966), 243-276. MR 34 \#626.

4. E. Hewitt and K. A. Ross, Abstract harmonic analysis. Vol. 1: Structure of topological groups. Integration theory, group representations, Die Grundlehren der math. Wissenschaften, Band 115, Academic Press, New York; Springer-Verlag, Berlin, 1963. MR 28 \#158.

5. B. E. Johnson, An introduction to the theory of centralizers, Proc. London Math. Soc. (3) 14 (1964), 299-320. MR 28 \#2450.

6. ——, Centralisers on certain topological algebras, J. London Math. Soc. 39 (1964), 603-614. MR 29 \#5115.

7. S. Mac Lane, Homology, Die Grundlehren der math. Wissenschaften, Band 114, Academic Press, New York; Springer-Verlag, Berlin, 1963. MR 28 \#122.

8. B. Mitchell, Theory of categories, Pure and Appl. Math., vol. 17, Academic Press, New York, 1965. MR 34 \#2647.

9. J. G. Wendel, Left centralizers and isomorphisms of group algebras, Pacific J. Math. 2 (1952), 251-261. MR 14, 246.

DREXEL UNIVERSITY, Philadelphia, Pennsylvania 19104 